SHORT REPORT

\title{
Clinical and molecular findings in IPEX syndrome
}

\author{
A K Myers, L Perroni, C Costigan, W Reardon
}

Arch Dis Child 2006;91:63-64. doi: 10.1136/adc.2005.078287

IPEX (immunodysregulation, polyendocrinopathy, enteropathy, $X$ linked syndrome) is a rare disorder which usually results in death in early infancy or childhood. Clinical awareness remains the cornerstone of diagnosis, and provided that the diagnosis is entertained, mutation analysis for FOXP3 gene mutations can be confirmatory. Two new patients in whom IPEX was diagnosed retrospectively are reported.

$\mathrm{P}$ rior to the recent designation as IPEX (immunodysregulation, polyendocrinopathy, enteropathy, $X$ linked) by the Human Genome Organisation (HUGO), this rare syndrome had been known by many alternative titles including $\mathrm{X}$ linked polyendocrinopathy, immune dysfunction, and diarrhoea (XPID), and X linked autoimmunity and allergic dysregulation (XLAAD). ${ }^{12}$ Published reports of this condition are limited and therefore the incidence is difficult to assess. ${ }^{2}$ Moreover the true incidence is probably higher than reflected by the current literature as it is likely that many cases have been under-reported or misdiagnosed. ${ }^{1}$ The clinical features which characterise the condition include early onset, insulin dependent, diabetes mellitus; chronic diarrhoea with villous atrophy; eczema; anaemia; thrombocytopenia; thyroiditis; and recurrent infections. Mutations of the FOXP3 gene cause the disease..$^{3-5}$

\section{CASE 1}

This boy was born at $35+1$ weeks' gestation by emergency caesarean section for severe intrauterine growth retardation (IUGR). Birth weight was $1370 \mathrm{~g}$ (<3rd centile). Hyperglycaemia was noted on day 1 , requiring treatment with intravenous insulin infusions. Blood sugars remained labile. Klebsiella sepsis was noted on day 19 of life when the patient became acutely unwell with hyperglycaemia, pyrexia, and irritability. Multiple investigations, including those for metabolic causation, were normal, as were cranial, renal, and pancreatic ultrasound scans.

An acute diarrhoeal illness at 5 weeks of age caused severe hypernatraemic dehydration, metabolic acidosis, renal impairment, and weight loss. A low free T4 but a normal TSH were recorded. A cardiorespiratory arrest occurred on say 54 of life. Postmortem examination revealed enteropathy, pancreatic inflammation and fibrosis, and lung pathology. Klebsiella was identified from culture tip at postmortem examination.

DNA analysis confirmed a sequence change at the FOXP3 gene. This sequence change $(1-7 G>T)$ (fig 1) was not previously described. It was not found in a control population of 200 males. Further testing on this patient's mother revealed that she was a carrier for this same change. As shown in fig 1 , the mutation is very likely to create a new ATG transcription initiation site with an abnormal reading frame, leading to a stop signal at codon 61 of the normal exon 1. The gene product thus generated will result in a truncated protein missing the functional forkhead winged helix domain.

\section{CASE 2}

This male infant was born at full term with a birth weight of $3.48 \mathrm{~kg}$. At age 4 days he developed fever, diarrhoea, and hyperglycaemia. He was also noted to be anaemic and dehydrated. Treatment with intravenous antibiotics and insulin ensued. Stool culture at this time was positive for Campylobacter jejunii infection. Other abnormalities emerged, specifically recurrent metabolic acidosis, poor urinary output, widespread dermatitis, and severe failure to thrive. Jejunal biopsy revealed absent villi and lymphocyte infiltration of the mucosa. Hospitalisation continued for over a year with recurrent episodes of sepsis, unstable diabetes, and unpredictable insulin needs. Recurrent infections and uncontrollable diarrhoea were major clinical challenges. Pseudomonas infection led to his demise. Postmortem findings revealed
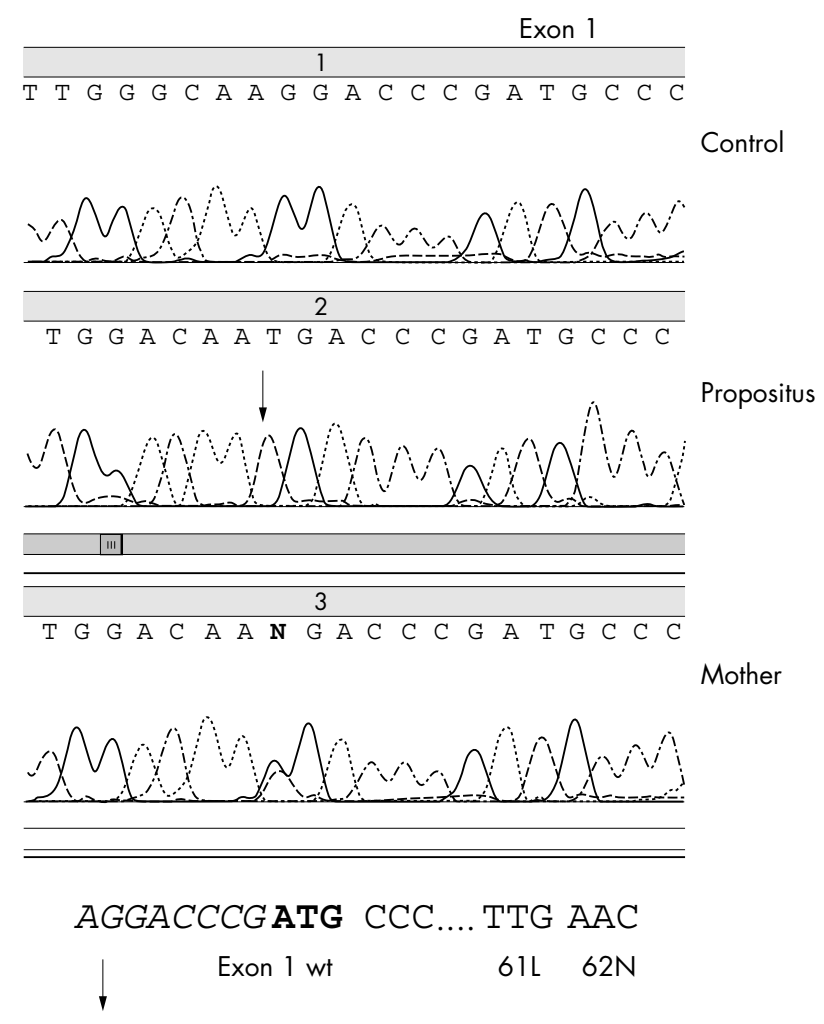

ATG ACC CGA TGC CCA....T TGA AC...

Exon 1 mutant stop

Figure 1 The $G>T$ substitution found in case 1 . At -7 from the canonical ATG starting codon a new ATG is created. The new reading frame creates a stop codon in exon 1, between codons 61 and 62. The mother of the propositus is heterozygous for the same mutation. 


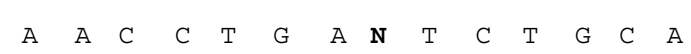

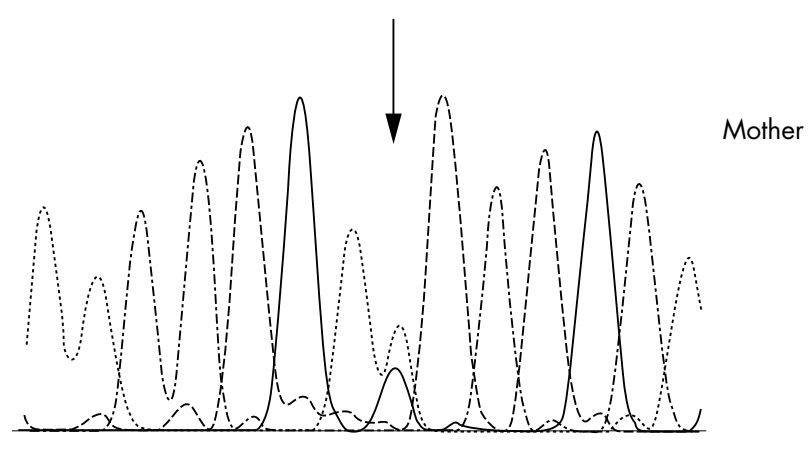

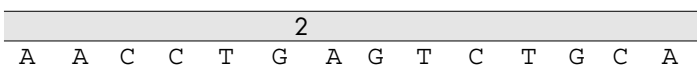

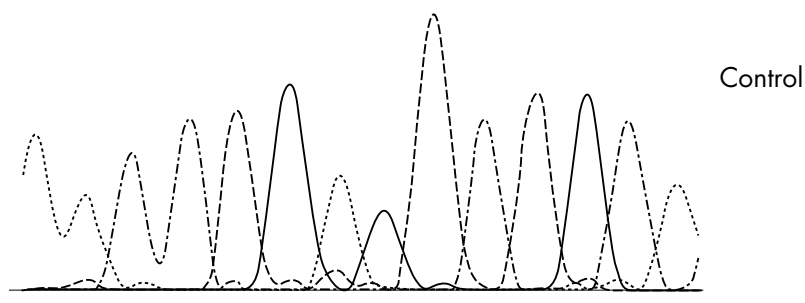

Figure 2 Electropherogram of exon 11 of the FOXP3 gene showing the $\mathrm{G}>\mathrm{A}$ substitution present at position 1169 in the mother of case 2 .

evidence of Pseudomonas septicaemia and suppurative otitis media together with suppurative myocarditis and pericarditis.

DNA was not available for analysis but, in view of the convincing history for IPEX syndrome, molecular DNA analysis of FOXP3 was carried out on his mother. A previously undescribed sequence alteration, G1169A was established in heterozygous form (fig 2). The predicted mutation (S390N) has not been previously described and was not found in a control population of 200 males. This mutation was absent in the normal brother of case 2, who had inherited the opposite maternal FOXP2 allele from his mother

\section{DISCUSSION}

The common phenotype found in classic IPEX is neonatal onset insulin dependent diabetes mellitus and an autoimmune enteropathy. Skin manifestations are also very common, in particular eczema and exfoliative or atopic dermatitis.

To date there have been 13 mutations of FOXP3 identified in association with individuals or pedigrees segregating IPEX. ${ }^{1}$ Most alter amino acid sequence in the forkhead winged helix DNA binding domain of the protein. The sequence change observed in the mother of case 2 in this report (S390N) is comparable to IPEX causing mutations in families with missense mutations in exons 9 or 10 of the FOXP3 gene. The likely pathogenicity of the mutation is enhanced by the observation that it is not present in the healthy brother of the proband, who inherited the opposite FOXP3 allele from his mother. Additionally, the serine at position 390 is a highly conserved amino acid, as are most of the amino acids of the functional domain of scurfin, an observation which also strongly supports likely pathogenicity.

The sequence change identified in case 1 of this report is especially interesting. A G to T substitution, seven base pairs upstream of the canonical AUG translation initiation site, results in the creation of a potentially competing initiation signal with new reading frame, resulting in a truncated protein for a stop signal at codon 61. Mutations causing upstream AUG sites are a rare but established mutational mechanism of disease, examples of which have been recorded at the POMC locus causing early onset obesity, adrenal insufficiency, and red hair pigmentation and also in beta thalassaemia intermedia. When the translation reading frame of the upstream AUG site is not the usual translation initiation site, as in the FOXP3 mutation reported here, translation beginning at this newly created site results in a protein sequence which bears no relation to the wild type.

We have identified likely pathogenic mutations in FOXP3 in the mothers of the two cases we present. These DNA alterations may, with the usual caveats, be used as the basis of a predictive test within the families concerned. The specific finding of a non-coding region mutation, causing the IPEX phenotype by creation of a false initiation site emphasises the need for immunologists to be aware of this particular mutational mechanism of disease and to specifically query DNA laboratories undertaking FOXP3 mutation analyses as to whether non-coding mutations are sought in the assays offered.

\section{ACKNOWLEDGEMENTS}

The authors wish to thank the families for their kind assistance and Dr Robert Wildin for much appreciated comments and direction. Dr Costigan wishes to acknowledge the input of Marie Carroll, Specialist Nurse in Paediatric Diabetology. Dr Reardon acknowledges support for his work from The Children's Medical and Research Foundation, Our Lady's Hospital for Sick Children.

\section{Authors' affiliations}

A K Myers, W Reardon, National Centre for Medical Genetics, Our Lady's Hospital for Sick Children, Crumlin, Dublin, Ireland L Perroni, Human Genetic Laboratory, Galliera Hospital, Via A. Volta 10, Genova 1612, Italy

C Costigan, Paediatric Endocrinology, Our Lady's Hospital for Sick Children, Crumlin, Dublin, Ireland

Competing interests: none declared

Correspondence to: Dr W Reardon, National Centre for Medical Genetics, Our Lady's Hospital for Sick Children, Crumlin, Dublin 12, Ireland; willie.reardon@olhsc.ie

Accepted 23 July 2005

\section{REFERENCES}

1 Wildin RS, Smyk-Pearson S, Filipovich AH. Clinical and molecular features of the immunodysregulation, polyendocrinopathy, enteropathy, $X$ linked (IPEX) syndrome. J Med Genet 2002;39:537-45.

2 Eisenbarth GS, Gottlieb PA. Autoimmune polyendocrine syndromes. N Engl J Med 2004;350:2068-77.

3 Wildin RS, Ramsdell F, Peake J, et al. X-linked neonatal diabetes mellitus, enteropathy and endocrinopathy syndrome of the mouse scurfy. Nature Genet 2001;27:18-20.

4 Bennett CL, Christie J, Ramsdell F, et al. The immune dysregulation, polyendocrinopathy, enteropathy, $x$-linked syndrome (IPEX) is caused by mutations of FOXP3. Nature Genet 2001;27:20-1.

5 Chatila TA, Blaeser F, Ho N, et al. JM2, encoding a fork head-related protein is mutated in X-linked autoimmunity-allergic dysregulation (sic) syndrome. $J$ Clin Invest 2000;106:R75-81. 